\title{
Robust Design of Two-Dimensional Optical Reference Signals Against Diffraction Effects
}

\author{
Yi-Sheng Su and Ozan K. Tonguz
}

\begin{abstract}
This paper presents two novel approaches to the design of two-dimensional (2-D) optical zero reference signals (ZRSs) that are robust against diffraction effects based on the parametric minimum cross-entropy (PMCE) method. Grating alignment systems require a 2-D optical ZRS to perform absolute measurements. A common method of acquiring 2-D optical ZRSs involves illuminating two identical superimposed 2-D zero reference codes (ZRCs). The output signal is the 2-D optical ZRS, and can be represented as the autocorrelation of the 2-D ZRC transmittance. In ultrahigh-resolution systems, diffraction distorts the shadow of the first 2-D ZRC, degrading the autocorrelation and greatly reducing the amplitude of the 2-D optical ZRS. To improve the robustness of 2-D optical ZRSs against diffraction effects, this paper formulates two combinatorial optimization problems for the design of 2-D ZRCs with minimum diffraction effects, one of which is a maximization problem, the other a minimization problem. Aiming at solving the two problems, this study proposes two schemes based on the PMCE method to search for an optimal 2-D ZRC. Simulation results reveal that there are $3.36 \% \sim 8.34 \%$ increases in the slope of the central peak of a 2-D optical ZRS and that there are $16.12 \% \sim 20.90 \%$ increases in the sum of the slope of the central peak and the effective signal amplitude of a 2-D optical ZRS, as compared with those obtained by the recently proposed cross-entropy (CE) method. The proposed PMCE-based schemes prove to search for 2-D ZRCs more effectively than existing solutions, while requiring less computational complexity.
\end{abstract}

Index Terms-Optical position measurement, optimization method, parametric minimum cross-entropy method.

\section{INTRODUCTION}

Alignment with submicron or even nanometer scale resolution is of vital importance in microelectronics, nanoscience and nanotechnology. The increasing demand for ultrahigh resolution in lithography, along with the reduction of the feature size of integrated circuits, has created a strong incentive for the design of grating alignment systems.

Grating alignment systems require an alignment signal, called the two-dimensional (2-D) optical zero reference signal (ZRS), to detect a predefined position. A common method of acquiring 2-D optical ZRSs consists of illuminating two identical superimposed 2-D zero reference codes (ZRCs). The optical output signal (to be registered in a photodetector) is the 2-D optical ZRS. 2-D ZRCs are binary transmittances which consist of a group of unevenly spaced opaque pixels on a transparent substrate. As one 2-D ZRC moves with respect to

This work has been supported by the National Science Council (NSC), Taiwan, ROC, under Contract NSC-100-2221-E-309-007.

Y.-S. Su is with the Department of Computer Science and Information Engineering, Chang Jung Christian University, Tainan, Taiwan, ROC.

O. K. Tonguz is with the Department of Electrical and Computer Engineering, Carnegie Mellon University, Pittsburgh, PA 15213-3890 USA. the other, it produces a change in the overlapping area between them, thereby producing a change in the transmitted light. When the codes stand opposite, the maximum of the signal is obtained and the two-axis alignment is achieved.

In recent years, several results [1]-[5] have been reported for the design of good 2-D ZRCs. In these reported results, the diffraction effects of the illuminating light are neglected as it propagates between the 2-D ZRCs, and the 2-D optical ZRS is the autocorrelation of the 2-D ZRCs. In this condition, called the "autocorrelation approximation," the design of 2-D ZRCs is modeled as an optimization problem of minimizing the second maximum of the autocorrelation.

Today, the requirements imposed by ultrahigh-resolution systems are making the use of very narrow pixels inevitable. This reduction in pixel size, in conjunction with the significant distance between the 2-D ZRCs, makes diffraction effects become much more noticeable. Diffraction distorts the shadow of the first 2-D ZRC onto the second one, and thus, the 2$\mathrm{D}$ optical ZRS becomes the correlation between the distorted shadow and the transmittance of the 2-D ZRC, invalidating the assumption of autocorrelation approximation. Diffraction has negative effects on a 2-D optical ZRS: decreasing the height of the central peak while increasing its width. Therefore, diffraction dramatically reduces the robustness and sensitivity of 2-D optical ZRSs [1], [6], [7], and renders the 2-D ZRCs obtained in [1]-[5] useless. To tackle the problem, SáezLandete et al. [7] calculated diffractive 2-D optical ZRSs using the angular spectrum of plane waves [8], proposed a design of 2-D ZRCs that accommodates diffraction effects and used a genetic algorithm (GA) to search for 2-D ZRCs. Motivated by the success of the cross-entropy (CE) method in solving complicated and large-scale combinatorial optimization problems, Chen et al. [9] recently applied the CE method to improve upon the design of [7]. Although the design in [9] can search for 2-D ZRCs for generating 2-D optical ZRSs less sensitive to diffraction effects, there is still room for improvement.

In contrast to [7], [9], this study formulates two combinatorial optimization problems for the design of 2-D ZRCs with minimum diffraction effects, one of which is a maximization problem, while the other a minimization problem. Aiming at solving the two problems, this study proposes two novel approaches based on the parametric minimum cross-entropy (PMCE) method to search for 2-D ZRCs with minimum diffraction effects. Simulation results show that the proposed approaches search for 2-D ZRCs more efficiently than the existing solutions, while requiring less computational complexity.

The rest of this paper is organized as follows. Section II 
describes the design problems in detail. Section III uses the PMCE method to design 2-D ZRCs with minimum diffraction effects. Section IV provides simulation results and discussion, and finally, Section V concludes the paper.

\section{PROBlEM Formulation}

As in [7], [9], this section first uses the angular spectrum approach to calculate diffractive 2-D optical ZRSs. Using the calculated results, this section then formulates two combinatorial optimization problems for the design of 2-D ZRCs with minimum diffraction effects.

\section{A. Calculation of Diffractive 2-D Optical ZRSs}

Fig. 1 shows the studied system for generating 2-D optical ZRSs. The system consists of two opposite identical 2-D ZRCs, at least one of which is set in an $x y$ movable stage. The 2-D ZRCs are separated by a distance $z$. The 2-D ZRC is a group of unequally spaced transparent and opaque pixels. The pixel size is denoted by $b$. To acquire a 2-D optical ZRS, a parallel ray beam propagates through both codes in the perpendicular direction. The optical output signal (to be registered in a photodetector) is the 2-D optical ZRS.

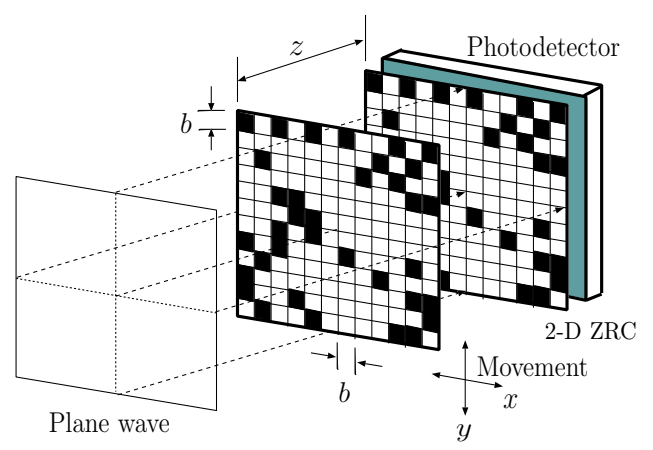

Fig. 1. System for generating 2-D optical ZRSs.

In general, a 2-D ZRC with $n \times n$ elements can be described by a square matrix $\mathbf{C}$ of binary data:

$\mathbf{C}=\left[\begin{array}{ccc}c_{11} & \cdots & c_{1 n} \\ \vdots & \ddots & \vdots \\ c_{n 1} & \cdots & c_{n n}\end{array}\right], \quad c_{i j} \in\{0,1\}, \quad 1 \leq i, j \leq n$,

where $c_{i j}=1$ if a transparent pixel is located at the $(i, j)$ position, and $c_{i j}=0$ elsewhere. Therefore, the transmittance of a 2-D ZRC $\mathbf{C}$ can be written as

$t_{\mathbf{C}}(x, y)=\sum_{i=1}^{n} \sum_{j=1}^{n} c_{i j} \operatorname{rect}\left(\frac{x-i \cdot b}{b}\right) \operatorname{rect}\left(\frac{y-j \cdot b}{b}\right)$,

where $\operatorname{rect}(\cdot)$ is the rectangle function defined as

$$
\operatorname{rect}(x)= \begin{cases}1, & \text { if }|x|<1 / 2 \\ 0, & \text { otherwise. }\end{cases}
$$

Assuming that the illuminating light is a parallel ray beam and that the thickness of the codes is negligible, the 2-D optical
ZRS can be calculated using the angular spectrum of plane waves [7], [8] as follows:

$$
S_{\mathbf{C}}(u, v)=\iint_{-\infty}^{+\infty}\left|U_{\mathbf{C}}(x, y)\right|^{2} t_{\mathbf{C}}(x+u, y+v) d x d y
$$

where $u$ and $v$ are the relative displacements between the 2-D ZRCs along the $x$ and $y$ directions of the codes, respectively, and $U_{\mathbf{C}}(x, y)$ is the field amplitude in a plane just before the second 2-D ZRC. The field amplitude $U_{\mathbf{C}}(x, y)$ can be expressed as

$$
\begin{aligned}
U_{\mathbf{C}}(x, y)=\iint T_{\mathbf{C}}(\nu, \eta) e^{j k z \sqrt{1-(\lambda \nu)^{2}-(\lambda \eta)^{2}}} & \\
& \times e^{j 2 \pi(\nu x+\eta y)} d \nu d \eta,
\end{aligned}
$$

where $T_{\mathbf{C}}(\nu, \eta)$ is the 2-D Fourier transform of the transmittance of the first 2-D ZRC in (1), $j=\sqrt{-1}, \lambda$ is the wavelength of the illuminating light, and $k=2 \pi / \lambda$ is the wave number. If $z=0$, i.e., no diffraction effects, then (3) implies $U_{\mathbf{C}}(x, y)=t_{\mathbf{C}}(x, y)$. Thus, (2) naturally becomes the autocorrelation approximation. The computation in (2) can be transformed into a 2-D convolution using the auxiliary transmittance $t_{\mathbf{C}}^{\prime}(x, y) \triangleq t_{\mathbf{C}}(-x,-y)$, and calculated in the frequency domain as follows:

$$
S_{\mathbf{C}}(u, v)=\operatorname{IFT} 2\left\{\mathrm{FT} 2\left\{\left|U_{\mathbf{C}}\right|^{2}\right\} \cdot \operatorname{FT} 2\left\{t_{\mathbf{C}}^{\prime}\right\}\right\},
$$

where FT2 and IFT2 are the direct and inverse 2-D Fourier transforms, respectively, and can be fulfilled by efficient 2-D fast Fourier transform (FFT) algorithms. For details, refer to [7].

\section{B. Combinatorial Optimization Problems}

Two of the most important parameters in grating alignment systems that characterize a 2-D optical ZRS are the "slope of the central peak" and the "effective signal amplitude" [7]. Taking into account the diffraction effects, the slope of the central peak of the 2-D optical ZRS generated by a 2-D ZRC $\mathbf{C}$ is measured by the following simple function:

$$
m_{1}(\mathbf{C})=S_{\mathbf{C}}(0,0)-\max _{\left\{(u, v) \mid u^{2}+v^{2}=(2 \cdot b)^{2}\right\}} S_{\mathbf{C}}(u, v),
$$

where $\left\{(u, v) \mid u^{2}+v^{2}=(2 \cdot b)^{2}\right\}$ is the set of points obtained when the relative displacement between the 2-D ZRCs is $2 \cdot b$. Clearly, $m_{1}(\mathbf{C})$ is the difference between the signal value when the 2-D ZRCs stand opposite, and the value when a relative displacement $2 \cdot b$ between the 2-D ZRCs takes place. In other words, the value of $m_{1}(\mathbf{C})$ is proportional to the slope between the signal maximum and the signal point when the relative displacement between the codes is $2 \cdot b$. On the other hand, the effective signal amplitude is the height difference between the first and second maxima, and is represented simply as

$$
m_{2}(\mathbf{C})=S_{\mathbf{C}}(0,0)-S_{\mathbf{C}}\left(u_{0}, v_{0}\right)
$$

where $S_{\mathbf{C}}\left(u_{0}, v_{0}\right)$ is the second maximum. ${ }^{1}$ Therefore, the larger the value of $m_{1}(\mathbf{C})$ or $m_{2}(\mathbf{C})$, the higher the robustness and sensitivity of a 2-D optical ZRS.

\footnotetext{
${ }^{1}$ The second maximum is the maximum signal value outside the central peak and can be obtained by searching the region outside the central peak.
} 
The number $n^{2}$ of pixels in a 2-D ZRC is typically limited by the diameter of the light beam propagating through both codes. In addition, in the absence of diffraction, the sensitivity of the photodetection optoelectronics determines the minimum value for the first maximum of the signal, i.e., the number $n_{1}$ of transparent pixels in a 2-D ZRC [1]-[5]. Thus, the numbers $n$ and $n_{1}$ are generally predetermined based on working requirements. Given the fixed sizes $n$ and $n_{1}$, it is then possible to generate 2-D optical ZRSs robust against diffraction effects by searching for 2-D optical ZRSs with a larger slope of the central peak and a higher effective signal amplitude. To achieve this, maximize

$$
m(\mathbf{C}) \triangleq m_{1}(\mathbf{C})+\mu \cdot m_{2}(\mathbf{C}),
$$

i.e., the weighted sum of $m_{1}(\mathbf{C})$ and $m_{2}(\mathbf{C})$, over the set of $n \times n$ binary matrices with $n_{1}$ ones in each, denoted by $\{0,1\}_{n_{1}}^{n \times n}$, where $\mu$ is the relative weight assigned to the maximization of the effective signal amplitude. ${ }^{2}$ A larger $\mu$ means that the effective signal amplitude weighs more in the weighted sum $m(\mathbf{C})$ and will possibly lead to a higher effective signal amplitude. In other words, the optimization problem can be stated as follows:

$$
\text { P1: } \quad \mathbf{C}^{*}=\arg \max _{\mathbf{C} \in\{0,1\}_{n_{1}}^{n \times n}} m(\mathbf{C}),
$$

where $\mathbf{C}^{*}$ denotes a 2-D ZRC attaining the global optimum of the weighted sum. Equivalently, we can minimize the reciprocal of the weighted sum, $m^{-1}(\mathbf{C}) \triangleq \frac{1}{m(\mathbf{C})}$, over $\{0,1\}_{n_{1}}^{n \times n}$ :

$$
\text { P2: } \quad \mathbf{C}^{*}=\arg \min _{\mathbf{C} \in\{0,1\}_{n 1}^{n \times n}} m^{-1}(\mathbf{C}) .
$$

The simplest way to solve Problem P1 or P2 is to search all possible $\mathbf{C}$ out of $\{0,1\}_{n_{1}}^{n \times n}$ to obtain an optimal 2D ZRC for generating a 2-D optical ZRS robust against diffraction effects. However, this approach leads to a total of $\left(\begin{array}{l}n^{2} \\ n_{1}\end{array}\right)$ possible combinations, which is a potential problem for practical implementation, especially for large codes. For example, in a typical design with $n=16$ and $n_{1}=148$, there are totally $2.53 \times 10^{74}$ combinations [7], [9]. To overcome this problem, two novel approaches to the design of 2-D ZRCs with minimum diffraction effects based on the PMCE method are proposed in the next section.

\section{Design Methodology}

This section employs the PMCE method to design 2-D ZRCs with minimum diffraction effects. The PMCE method was first proposed by Rubinstein and Dolgin [10] for solving rare event probability estimation and counting problems. Similar to the CE method, the PMCE method first associates the original deterministic optimization problem with a relevant rare-event probability estimation, and then finds the optimal parameters of a probability distribution to efficiently estimate the desired quantity. However, unlike the $\mathrm{CE}$ method that derives the optimal parameters of the probability distribution

\footnotetext{
${ }^{2}$ The weighted sum $m(\mathbf{C})$ includes as a special case the objective function in [7], [9], which corresponds to the case with $\mu=0$, i.e., $m(\mathbf{C})=m_{1}(\mathbf{C})$.
}

from the CE program, the PMCE method uses the parametric version of the minimum cross-entropy program to obtain the optimal parameters of the probability distribution. For details of the PMCE method, refer to [10].

\section{A. Solving Problem P1}

The first step to solve the deterministic optimization Problem P1 in (8) using the PMCE method is to associate it with a meaningful estimation problem. To this end, the $(i$, $j$ ) element of a 2-D ZRC $\mathbf{C}$ is modeled as an independent Bernoulli random variable with the probability mass function $\operatorname{Pr}\left\{c_{i j}=1\right\}=p_{i j}, \operatorname{Pr}\left\{c_{i j}=0\right\}=1-p_{i j}, 1 \leq i, j \leq n$. Accordingly, the probability density function of $\mathbf{C}$ is

$$
f(\mathbf{C} ; \mathbf{P})=\prod_{i=1}^{n} \prod_{j=1}^{n} p_{i j}^{c_{i j}}\left(1-p_{i j}\right)^{1-c_{i j}}
$$

where

$$
\mathbf{P}=\left[\begin{array}{ccc}
p_{11} & \cdots & p_{1 n} \\
\vdots & \ddots & \vdots \\
p_{n 1} & \cdots & p_{n n}
\end{array}\right]
$$

Then, associate (8) with the problem of estimating the probability of the event $\{m(\mathbf{C}) \geq \gamma\}$ for a given threshold $\gamma$. For a sufficiently large value of $\gamma$, e.g., $\gamma=m\left(\mathbf{C}^{*}\right)$, $\operatorname{Pr}\{m(\mathbf{C}) \geq \gamma\}$ is a rare-event probability. To estimate $\operatorname{Pr}\{m(\mathbf{C}) \geq \gamma\}$ efficiently, the PMCE method iteratively updates the probability matrix $\mathbf{P}$ so that most random samples generated according to $f(\mathbf{C} ; \mathbf{P})$ in (10) satisfy $m(\mathbf{C}) \geq \gamma$. Meanwhile, the PMCE method also iteratively improves $\gamma$ so that $f(\mathbf{C} ; \mathbf{P})$ eventually converges to an optimal probability distribution $f\left(\mathbf{C} ; \mathbf{P}^{*}\right)$ that generates an optimal $\mathbf{C}^{*}$, and $\gamma$ then converges to the optimal $\gamma^{*}$. Specifically, the updating procedures are detailed as follows:

1) Adaptive updating of $\gamma^{l}$ : For a fixed $\mathbf{P}^{l-1}$, generate $Q$ random samples $\mathbf{C}_{1}^{l}, \ldots, \mathbf{C}_{Q}^{l}$ from $f\left(\cdot ; \mathbf{P}^{l-1}\right)$, where $l$ denotes the iteration index. Here, each sample $\mathbf{C}_{q}^{l}, 1 \leq$ $q \leq Q$, is an $n \times n$ binary matrix with $n_{1}$ ones in it, representing a 2-D ZRC. Then, calculate the weighted sum $m\left(\mathbf{C}_{q}^{l}\right)$ for all $q$ and sort them from largest to smallest such that

$$
m\left(\mathbf{C}_{(1)}^{l}\right) \geq \cdots \geq m\left(\mathbf{C}_{(Q)}^{l}\right) .
$$

Finally, set

$$
\gamma^{l}=\frac{1}{\lceil\rho Q\rceil} \sum_{q=1}^{\lceil\rho Q\rceil} m\left(\mathbf{C}_{(q)}^{l}\right),
$$

where $\rho \in(0,1)$ is called the rarity parameter [10] and $\lceil\cdot\rceil$ is the ceiling function.

2) Adaptive updating of $\mathbf{P}^{l}$ : For fixed $\gamma^{l}$ and $\mathbf{P}^{l-1}$, use the samples $\mathbf{C}_{1}^{l}, \ldots, \mathbf{C}_{Q}^{l}$ to update the parameter

$$
\mathbf{P}^{l}=\left[\begin{array}{ccc}
p_{11}^{l} & \cdots & p_{1 n}^{l} \\
\vdots & \ddots & \vdots \\
p_{n 1}^{l} & \cdots & p_{n n}^{l}
\end{array}\right]
$$


with

$$
\begin{array}{r}
p_{i j}^{l}=\frac{\sum_{q=1}^{Q} \mathbb{I}_{\left\{c_{q, i j}^{l}=1\right\}} \exp \left(-m\left(\mathbf{C}_{q}^{l}\right) \beta^{l}\right)}{\sum_{q=1}^{Q} \exp \left(-m\left(\mathbf{C}_{q}^{l}\right) \beta^{l}\right)}, \\
1 \leq i, j \leq n,
\end{array}
$$

where $\mathbb{I}_{\{\cdot\}}$ is the indicator of an event $\{\cdot\}, c_{q, i j}^{l}$ denotes the $(i, j)$ element of $\mathbf{C}_{q}^{l}, 1 \leq q \leq Q$, and the parameter $\beta^{l}$ is obtained from the solution of the following equation:

$$
\gamma^{l}=\frac{\sum_{q=1}^{Q} m\left(\mathbf{C}_{q}^{l}\right) \exp \left(-m\left(\mathbf{C}_{q}^{l}\right) \beta^{l}\right)}{\sum_{q=1}^{Q} \exp \left(-m\left(\mathbf{C}_{q}^{l}\right) \beta^{l}\right)} .
$$

In practice, to prevent a fast convergence to a local optimum, $\mathbf{P}^{l-1}$ is not updated directly to $\mathbf{P}^{l}$ via (13). Instead, a smoothing parameter $\alpha$ is introduced [10] to update (13) smoothly via

$$
\mathbf{P}^{l}=\alpha \mathbf{P}^{l}+(1-\alpha) \mathbf{P}^{l-1},
$$

where $\alpha \in(0,1]$. Clearly, when $\alpha=1$, the original updating formula is obtained.

Given the fixed sizes $n$ and $n_{1}$, the proposed PMCEbased scheme for solving Problem P1, called PMCE 1, aims at finding an $n \times n$ binary matrix $\mathbf{C}^{*}$ with $n_{1}$ ones in it, maximizing the weighted sum in (8), and is summarized as follows.

PMCE 1:

1) Set $l=1$ and initialize the probability matrix

$$
\mathbf{P}^{0}=\left[\begin{array}{ccc}
1 / 2 & \cdots & 1 / 2 \\
\vdots & \ddots & \vdots \\
1 / 2 & \cdots & 1 / 2
\end{array}\right]
$$

2) Randomly generate $Q$ samples $\mathbf{C}_{1}^{l}, \ldots, \mathbf{C}_{Q}^{l}$ according to $f\left(\cdot ; \mathbf{P}^{l-1}\right)$.

3) Compute the weighted sum $m\left(\mathbf{C}_{q}^{l}\right)$ for $q=1, \ldots, Q$ and use (12) to update $\gamma^{l}$.

4) Evaluate $\mathbf{P}^{l}$ via (13) and then update $\mathbf{P}^{l}$ smoothly via (15).

5) Output the optimal $\mathbf{C}^{*}$ upon reaching the predefined number $L$ of iterations. Otherwise, increase $l$ by 1 and return to Step 2.

\section{Remarks:}

- In Step 2, it may be necessary to randomly add/remove ones to/from the generated samples to meet the constraint on the number $n_{1}$ of transparent pixels in a 2-D ZRC.

- In Step 4, the entire sample size is used in (13), which is in contrast to the CE method where only the elite sample is used.

- Note that (13) is similar to the standard CE formula (16) in [9] with only one difference: the indicator function $\mathbb{I}_{\left\{m\left(\mathbf{C}_{q}^{l}\right) \geq \gamma^{l}\right\}}$ in the CE update formula is replaced here by $\exp \left(-m\left(\mathbf{C}_{q}^{l}\right) \beta^{l}\right)$. Thus, in the CE method, $p_{i j}^{l}$ is the fraction of samples in the elite-sample set with the $(i, j)$ element equal to one at iteration $l$. This indicates that in the CE method, given the elite-sample set at iteration $l, p_{i j}^{l}$ is independent of the values of the weighted sum,
$m\left(\mathbf{C}_{q}^{l}\right), 1 \leq q \leq Q$. However, in the PMCE method, the updating formula of $p_{i j}^{l}$ in (13) depends directly on the values of the weighted sum due to the function $\exp \left(-m\left(\mathbf{C}_{q}^{l}\right) \beta^{l}\right)$. This motivates the use of the PMCE method to solve the equivalent Problem P2 with the hope that better 2-D ZRCs can be obtained, as the objective function of Problem P2 in (9), i.e., the reciprocal of the weighted sum, is different from that of Problem P1 in (8).

\section{B. Solving Problem P2}

Similarly to solving Problem P1, associate (9) with an estimation problem: estimating the probability of the event $\left\{m^{-1}(\mathbf{C}) \leq \gamma\right\}$ for a given threshold $\gamma$. For a sufficiently small value of $\gamma$, e.g., $\gamma=m^{-1}\left(\mathbf{C}^{*}\right), \operatorname{Pr}\left\{m^{-1}(\mathbf{C}) \leq \gamma\right\}$ is a rare-event probability. To estimate $\operatorname{Pr}\left\{m^{-1}(\mathbf{C}) \leq \gamma\right\}$ efficiently, the PMCE method iteratively updates the probability matrix $\mathbf{P}$ and $\gamma$. The updating procedures for solving Problem $\mathrm{P} 2$ are exactly the same as those for solving Problem P1 except that (11), (12), (13), and (14) are replaced, respectively, by

$$
\begin{gathered}
m^{-1}\left(\mathbf{C}_{(1)}^{l}\right) \leq \cdots \leq m^{-1}\left(\mathbf{C}_{(Q)}^{l}\right), \\
\gamma^{l}=\frac{1}{\lceil\rho Q\rceil} \sum_{q=1}^{\lceil\rho Q\rceil} m^{-1}\left(\mathbf{C}_{(q)}^{l}\right)
\end{gathered}
$$

$$
\begin{array}{r}
p_{i j}^{l}=\frac{\sum_{q=1}^{Q} \mathbb{I}_{\left\{c_{q, i j}^{l}=1\right\}} \exp \left(-m^{-1}\left(\mathbf{C}_{q}^{l}\right) \beta^{l}\right)}{\sum_{q=1}^{Q} \exp \left(-m^{-1}\left(\mathbf{C}_{q}^{l}\right) \beta^{l}\right)}, \\
1 \leq i, j \leq n,
\end{array}
$$

and

$$
\gamma^{l}=\frac{\sum_{q=1}^{Q} m^{-1}\left(\mathbf{C}_{q}^{l}\right) \exp \left(-m^{-1}\left(\mathbf{C}_{q}^{l}\right) \beta^{l}\right)}{\sum_{q=1}^{Q} \exp \left(-m^{-1}\left(\mathbf{C}_{q}^{l}\right) \beta^{l}\right)}
$$

Given the fixed sizes $n$ and $n_{1}$, the proposed PMCEbased scheme for solving Problem P2, called PMCE 2, aims at finding an $n \times n$ binary matrix $\mathbf{C}^{*}$ with $n_{1}$ ones in it, minimizing the reciprocal of the weighted sum in (9), and is exactly the same as PMCE 1 in Section III-A except that Step 3 and Step 4 are replaced, respectively, by the following Step 3' and Step 4':

3') Compute the reciprocal of the weighted sum $m^{-1}\left(\mathbf{C}_{q}^{l}\right)$ for $q=1, \ldots, Q$ and use (17) to update $\gamma^{l}$.

$\left.4^{\prime}\right)$ Evaluate $\mathbf{P}^{l}$ via (18) and then update $\mathbf{P}^{l}$ smoothly via (15).

\section{REsults And Discussion}

For comparison with [9], this study considers a grating alignment system with the following settings: the number of pixels in the 2-D ZRCs is $n^{2}=16^{2}$, the size of a pixel is $b=10 \mu \mathrm{m}$, the distance between the 2-D ZRCs is $z=400$ $\mu \mathrm{m}$, and the wavelength used is $\lambda=635 \mathrm{~nm}$. In addition, this study considers two cases with different constraints on the number $n_{1}$ of transparent pixels in the 2-D ZRCs: Case 1 
$\left(n_{1}=100\right)$ and Case $2\left(n_{1}=148\right) .^{3}$ As in [9], the proposed PMCE-based schemes are run with $\rho=0.1, \alpha=0.7$, $Q=300$ and $L=40$. However, in contrast to [9], where 100 independent trials are performed, this study conducts only 50 independent trials for the proposed schemes. For simplicity, the results designed with the GA-based [7] and CE-based [9] schemes are labeled as "GA" and "CE," respectively. This study uses 2-D FFT algorithms to facilitate the computation of (4). Some details regarding the computation of (4) are in order: 1) a rectangular window function is used for the windowing of the FFT computation, 2) the sampling interval in the spatial domain is $1 \mu \mathrm{m}, 3)$ the sampling interval in the frequency domain is the reciprocal of that in the spatial domain, and 4) suitable zero-padding is needed for accurate results. The results below are normalized to the maximum of the autocorrelation.

\section{A. Design With $\mu=0$}

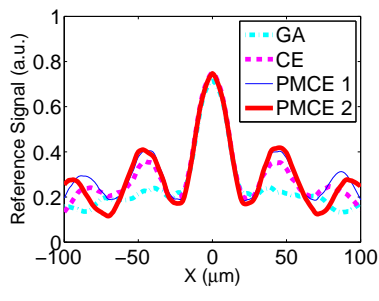

(a)

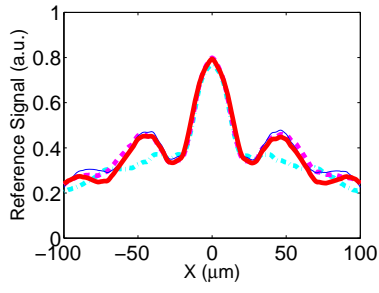

(b)
Fig. 2. Comparison of the central profiles of the 2-D optical ZRSs designed optimally with $\mu=0$ (a.u.: arbitrary units): (a) Case 1; (b) Case 2.

With $\mu=0$, i.e., under the objective function in [7], [9], Fig. 2 depicts the central profiles of the 2-D optical ZRSs obtained with different 2-D ZRCs that are chosen optimally from the trials. Fig. 2 shows that the signals all have one single central peak. For a more accurate comparison, Table I shows the quantities of the weighted sum $m(\mathbf{C})$ and the effective signal amplitude $m_{2}(\mathbf{C})$ for different signals. This table shows that the signals designed with PMCE 1 and PMCE 2 have a larger $m(\mathbf{C})$ than those designed with GA or CE. More specifically, PMCE 1 provides $7.89 \%$ and $3.36 \%$ increases in $m(\mathbf{C})$ for Case 1 and Case 2, respectively, while PMCE 2 provides $8.34 \%$ and $3.46 \%$ increases for the same cases, as compared to that of $\mathrm{CE}$. In addition, the table also shows that PMCE 2 performs better than PMCE 1. This is achieved at the expense of additional computation of reciprocal operations. Because the search result in each independent trial is not invariably a global optimum, Table II shows the averages (AVGs) and standard deviations (SDs) of $m(\mathbf{C})$ and $m_{2}(\mathbf{C})$ obtained in the trials for PMCE 1 and PMCE 2. Comparing Table II with Table I reveals that the AVGs of $m(\mathbf{C})$ for PMCE 1 and PMCE 2 are higher than the optimal values of $m(\mathbf{C})$ for GA and CE. This indicates that PMCE 1 and PMCE 2 must have higher AVGs of $m(\mathbf{C})$ than GA and CE,

\footnotetext{
${ }^{3}$ Four cases are studied in [9], but due to their similarities and for presentation simplicity, only two of them are considered in this study.
}

and hence, the PMCE-based schemes are superior to both the GA-based and CE-based schemes. Table II also shows that PMCE 2 has a larger SD of $m(\mathbf{C})$ than PMCE 1, although PMCE 2 outperforms PMCE 1.

TABLE I

QUANTITATIVE COMPARISON BETWEEN DIFFERENT 2-D OPTICAL ZRSS DESIGNED OPTIMALLY WITH $\mu=0$

\begin{tabular}{c||c||c||c||c||c}
\hline \hline & Case & GA & CE & PMCE 1 & PMCE 2 \\
\hline \hline \multirow{2}{*}{$m(\mathbf{C})$} & 1 & 0.4650 & 0.5071 & 0.5471 & 0.5494 \\
\cline { 2 - 6 } & 2 & 0.3897 & 0.4102 & 0.4240 & 0.4244 \\
\hline \hline \multirow{2}{*}{$m_{2}(\mathbf{C})$} & 1 & 0.4253 & 0.3444 & 0.3369 & 0.2905 \\
\cline { 2 - 6 } & 2 & 0.3265 & 0.3384 & 0.3178 & 0.3420 \\
\hline \hline
\end{tabular}

TABLE II

AVERAGES (AVGS) AND STANDARD DEVIATIONS (SDS) OF $m(\mathbf{C})$ AND $m_{2}(\mathbf{C})$ FOR DIFFERENT 2-D OPTICAL ZRSS DESIGNED WITH $\mu=0$

\begin{tabular}{c||c||c||c||c||c}
\hline \multicolumn{1}{c||}{} & \multirow{2}{*}{ Case } & \multicolumn{2}{c||}{ PMCE 1 } & \multicolumn{2}{c}{ PMCE 2 } \\
\cline { 3 - 6 } & & AVG & SD & AVG & SD \\
\hline \hline \multirow{2}{*}{$m(\mathbf{C})$} & 1 & 0.5206 & 0.0112 & 0.5212 & 0.0131 \\
\cline { 2 - 6 } & 2 & 0.4110 & 0.0067 & 0.4116 & 0.0069 \\
\hline \hline \multirow{2}{*}{$m_{2}(\mathbf{C})$} & 1 & 0.3681 & 0.0311 & 0.3693 & 0.0361 \\
\cline { 2 - 6 } & 2 & 0.3280 & 0.0237 & 0.3275 & 0.0266 \\
\hline \hline
\end{tabular}

Figs. 3 and 4 plot the 2-D optical ZRSs designed optimally with PMCE 1 and PMCE 2, respectively, where the diffractive effects are considered. Figs. 3 and 4 show that the signals may seem to be symmetric with respect to the origin. But, 2-D optical ZRSs generally do not have origin symmetry, especially when considering diffraction effects. This is due to the function $e^{j k z \sqrt{1-(\lambda \nu)^{2}-(\lambda \eta)^{2}}}$ in (3). Figs. 5 and 6 depict the 2-D ZRCs designed for generating the signals shown in Figs. 3 and 4, respectively, where each white square represents a transparent pixel. Figs. 5 and 6 show that the 2-D ZRCs have the transparent pixels grouped. This is because grouped pixels involve a fewer number of hard edges in the transmittance, leading to less high frequency content in its Fourier spectrum and weaker diffraction effects.

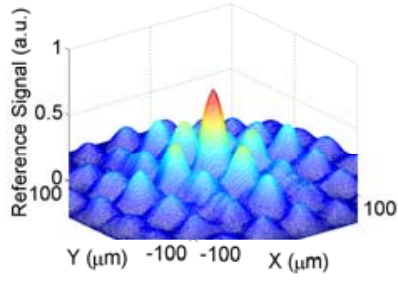

(a)

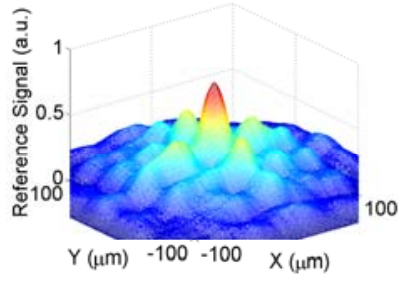

(b)
Fig. 3. 2-D optical ZRSs obtained with the 2-D ZRCs designed optimally with $\mu=0$ and PMCE 1: (a) Case 1; (b) Case 2.

The 2-D ZRCs shown in Figs. 5 and 6 are designed according to fixed design parameters $b, z$ and $\lambda$. In practice, the actual values of these design parameters may vary from the setting ones. To investigate the robustness of the signals against the variations in the design parameters, this 


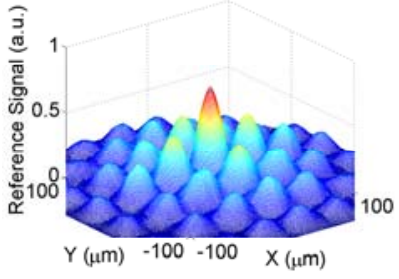

(a)

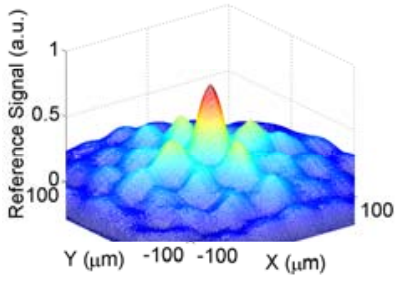

(b)
Fig. 4. 2-D optical ZRSs obtained with the 2-D ZRCs designed optimally with $\mu=0$ and PMCE 2: (a) Case 1; (b) Case 2.

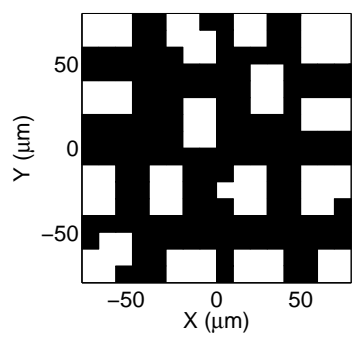

(a)

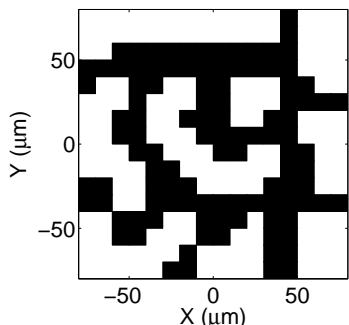

(b)
Fig. 5. 2-D ZRCs designed optimally with $\mu=0$ and PMCE 1: (a) Case 1 ; (b) Case 2.

study varies one of the design parameters while keeping the remaining two fixed. Due to similarity of results, this study only presents partial results obtained by varying $b$. Fig. 7 plots the central profiles of the signals obtained with the 2-D ZRCs shown in Fig. 5, where $b$ varies from 10 to $15 \mu \mathrm{m}$ with step size $1 \mu \mathrm{m}$. Fig. 7 shows that even though the actual parameter is not matched to the design value, the signals still perform well. With the goal of including parameter variations in the design process while maintaining robustness, this observation motivates to simply modify the definitions of $b, z$ and $\lambda$ as the minimum pixel size, the maximum distance between codes and the maximum wavelength of the illuminating light, respectively. The rationale behind the modification is explained as follows. Diffraction effects are negligible when $b \gg z$ and $b \gg \lambda$. To make diffraction effects become unnoticeable, it is intuitively desirable to have $b$ as large as possible, $z$ as small

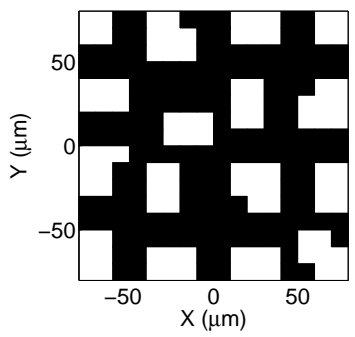

(a)

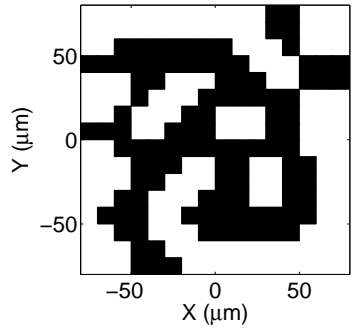

(b)
Fig. 6. 2-D ZRCs designed optimally with $\mu=0$ and PMCE 2: (a) Case 1; (b) Case 2. as possible and $\lambda$ as small as possible. Hence, if the initial conditions of $b, z$ and $\lambda$ can be chosen conservatively, the signals have a bit better robustness against the variations in the design parameters. In addition, Fig. 7 also shows that the signals designed with a large $b$ have not only a wider width at the base (low resolution), but also a higher central peak (less diffraction). This demonstrates that there is a tradeoff between the resolution of the system and diffraction effects when varying $b$.

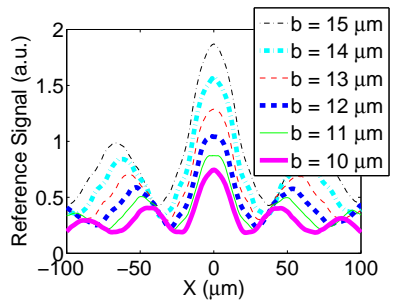

(a)

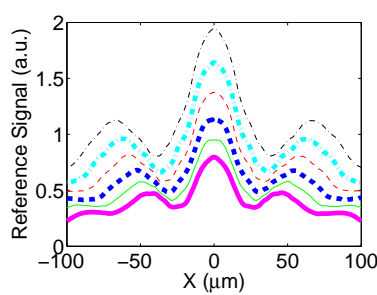

(b)
Fig. 7. Central profiles of the 2-D optical ZRSs obtained with the 2-D ZRCs shown in Fig. 5 under different values of $b$ : (a) Case 1; (b) Case 2.

Because the proposed schemes are iterative algorithms, convergence is of paramount importance. To demonstrate the convergence of the schemes, Fig. 8 plots the best objective function value in each iteration for the design of the 2-D ZRCs shown in Figs. 5 and 6. Fig. 8 shows that the best objective function value roughly improves with increasing iteration number and that the number of iterations required to reach the convergence is about 40 .

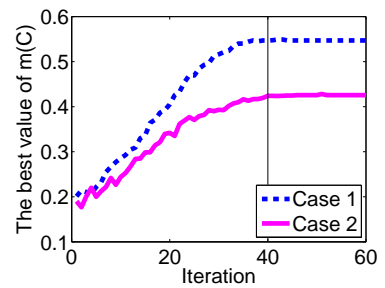

(a)

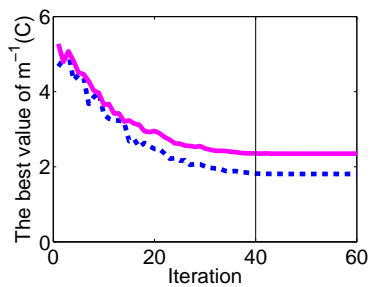

(b)
Fig. 8. The best objective function value in each iteration for the design of the 2-D ZRCs shown in (a) Fig. 5 and (b) Fig. 6.

This study compares the total number of samples generated with respect to computational complexity, as the design method are population-based search approaches. The total number of samples generated by each of the two proposed schemes is equal to $50 \times 40 \times 300=600,000$, which is only $50 \%$ of that generated by CE in [9], $100 \times 40 \times 300=$ 1, 200, 000 samples. $^{4}$

\footnotetext{
${ }^{4}$ One might think that the comparison of computational complexity in terms of the total number of samples generated is unfair for CE in [9], as an iteration of the proposed schemes may be more expensive than that of CE. However, as the number $n^{2}$ of pixels in a 2-D ZRC increases, such a comparison seems rather justified. This is because as $n^{2}$ increases, the total amount of computation is dominated by the calculation of 2-D optical ZRSs $S_{\mathbf{C}}(u, v)$ in (2).
} 


\section{B. Design With $\mu=1$}

As seen in Figs. 2, 3 and 4, one major drawback of the 2-D optical ZRSs designed with the PMCE-based schemes in Section IV-A is their high second maxima, possibly resulting in a lower effective signal amplitude. This is primarily because with $\mu=0$, (8) or (9) does not account for the optimization of the effective signal amplitude. To optimize the effective signal amplitude of the 2-D optical ZRSs, increase $\mu$ to $1 .{ }^{5}$ With $\mu=1$, Fig. 9 depicts the central profiles of the 2-D optical ZRSs obtained with different 2-D ZRCs that are chosen optimally. Fig. 9 shows that the 2-D optical ZRSs designed with $\mu=1$ have a relatively flat region outside the central peak compared with Fig. 2. For a more accurate comparison, Tables III and IV show the statistical quantities of $m(\mathbf{C})$, $m_{1}(\mathbf{C})$ and $m_{2}(\mathbf{C})$ for different 2-D optical ZRSs obtained in the trials. In particular, Table III shows that with $\mu=1$, PMCE 1 provides $20.43 \%$ and $16.46 \%$ increases in $m(\mathbf{C})$ for Case 1 and Case 2, respectively, while PMCE 2 provides $20.90 \%$ and $16.12 \%$ increases for the same cases, as compared to that of CE. Table III also shows that with $\mu=1,1)$ the signals designed with PMCE 1 and PMCE 2 no longer have a larger $m_{1}(\mathbf{C})$ than those designed with GA or CE, and 2) $m_{1}(\mathbf{C})$ decreases, whereas $m_{2}(\mathbf{C})$ increases, as compared to the design with $\mu=0$. This is because with $\mu=1, m_{2}(\mathbf{C})$ weighs more in the weighted sum compared to the design with $\mu=0$. Interestingly, Tables III shows that with $\mu=1$, the signal designed with $\mathrm{CE}$ has a slightly smaller $m(\mathbf{C})$ than that designed with GA for Case 1.

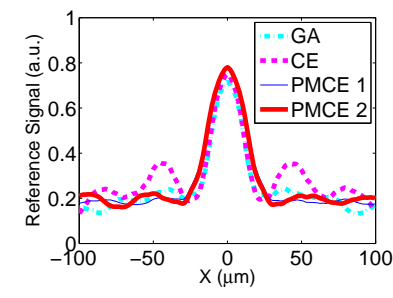

(a)

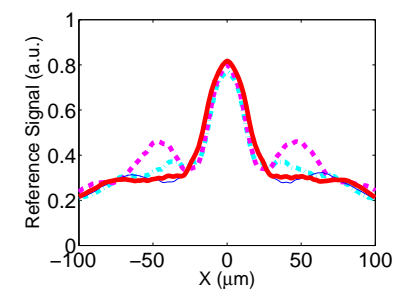

(b)
Fig. 9. Comparison of the central profiles of the 2-D optical ZRSs designed optimally with $\mu=1$ : (a) Case 1; (b) Case 2.

TABLE III

QUANTITATIVE COMPARISON BETWEEN DIFFERENT 2-D OPTICAL ZRSS DESIGNED OPTIMALLY WITH $\mu=1$

\begin{tabular}{c||c||c||c||c||c}
\hline \hline & Case & GA & CE & PMCE 1 & PMCE 2 \\
\hline \hline \multirow{2}{*}{$m(\mathbf{C})$} & 1 & 0.8903 & 0.8515 & 1.0255 & 1.0295 \\
\cline { 2 - 6 } & 2 & 0.7162 & 0.7486 & 0.8718 & 0.8693 \\
\hline \hline \multirow{2}{*}{$m_{1}(\mathbf{C})$} & 1 & 0.4650 & 0.5071 & 0.4597 & 0.4708 \\
\cline { 2 - 6 } & 2 & 0.3897 & 0.4102 & 0.3828 & 0.3779 \\
\hline \hline \multirow{2}{*}{$m_{2}(\mathbf{C})$} & 1 & 0.4253 & 0.3444 & 0.5658 & 0.5587 \\
\cline { 2 - 6 } & 2 & 0.3265 & 0.3384 & 0.4890 & 0.4914 \\
\hline \hline
\end{tabular}

\footnotetext{
${ }^{5}$ This study considers several different values of $\mu$ but finds that $\mu=$ 1 provides the best tradeoff between the slope of the central peak and the effective signal amplitude.
}

TABLE IV

AVGs AND SDS OF $m(\mathbf{C}), m_{1}(\mathbf{C})$ AND $m_{2}(\mathbf{C})$ FOR DIFFERENT 2 -D OPTICAL ZRSS DESIGNED WITH $\mu=1$

\begin{tabular}{|c|c|c|c|c|c|}
\hline & \multirow{2}{*}{ Case } & \multicolumn{2}{|c|}{ PMCE 1} & \multicolumn{2}{|c|}{ PMCE 2} \\
\hline & & AVG & SD & AVG & SD \\
\hline \multirow{2}{*}{$m(\mathbf{C})$} & 1 & 0.9949 & 0.0148 & 0.9961 & 0.0156 \\
\hline & 2 & 0.8462 & 0.0125 & 0.8457 & 0.0123 \\
\hline \multirow{2}{*}{$m_{1}(\mathbf{C})$} & 1 & 0.5352 & 0.0159 & 0.5342 & 0.0160 \\
\hline & 2 & 0.4722 & 0.0104 & 0.4714 & 0.0110 \\
\hline \multirow{2}{*}{$m_{2}(\mathbf{C})$} & 1 & 0.4596 & 0.0114 & 0.4619 & 0.0125 \\
\hline & 2 & 0.3739 & 0.0071 & 0.3743 & 0.0069 \\
\hline
\end{tabular}

Figs. 10 and 11 plot the 2-D optical ZRSs obtained with the 2-D ZRCs designed optimally with PMCE 1 and PMCE 2, respectively, where the diffractive effects are considered. The corresponding 2-D ZRCs designed for generating the signals are depicted in Figs. 12 and 13, respectively. Figs. 10 and 11 show that the signals have a flatter region outside the central peak than those in Figs. 3 and 4.

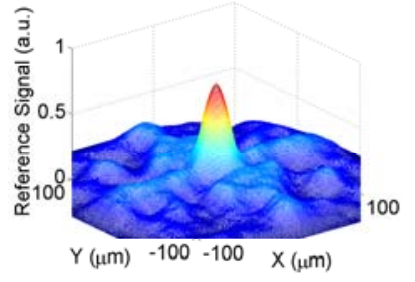

(a)

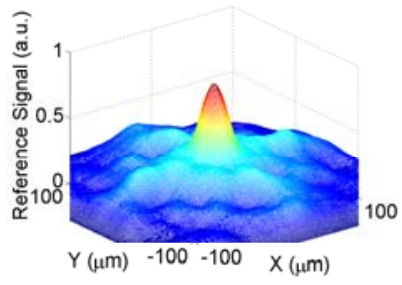

(b)
Fig. 10. 2-D optical ZRSs obtained with the 2-D ZRCs designed optimally with $\mu=1$ and PMCE 1: (a) Case 1; (b) Case 2.

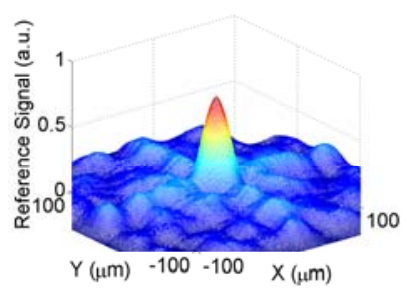

(a)

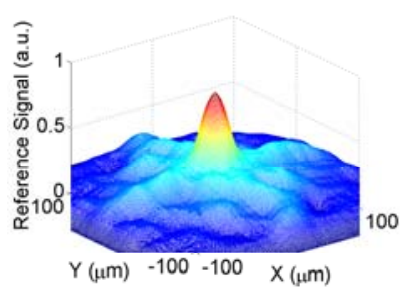

(b)
Fig. 11. 2-D optical ZRSs obtained with the 2-D ZRCs designed optimally with $\mu=1$ and PMCE 2: (a) Case 1; (b) Case 2.

\section{CONCLUSION}

This paper proposes two PMCE-based schemes that can be used to search for an optimal 2-D ZRC with minimum diffraction effects. Simulation results show that the two schemes search for 2-D ZRCs more effectively than existing methods, while requiring less computational complexity. More specifically, it is observed that there are $3.36 \% \sim 8.34 \%$ increases in the slope of the central peak of a 2-D optical ZRS and that there are $16.12 \% \sim 20.90 \%$ increases in the sum of the slope of the central peak and the effective signal amplitude of 


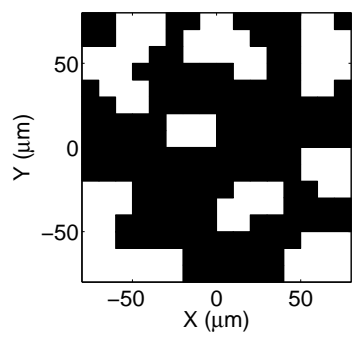

(a)

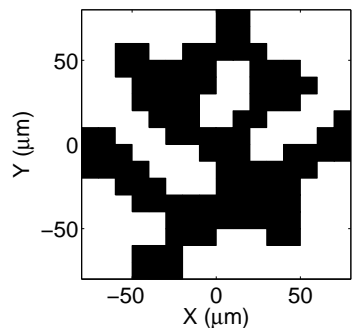

(b)

Fig. 12. 2-D ZRCs designed optimally with $\mu=1$ and PMCE 1: (a) Case 1 ; (b) Case 2.

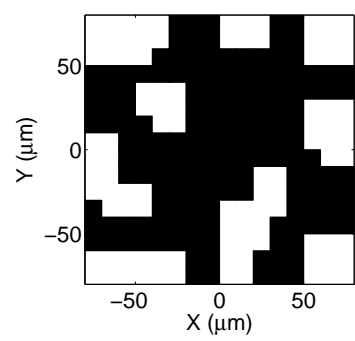

(a)

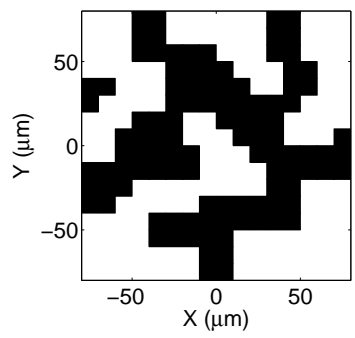

(b)

Fig. 13. 2-D ZRCs designed optimally with $\mu=1$ and PMCE 2: (a) Case 1 ; (b) Case 2.

a 2-D optical ZRS, as compared with those obtained by the CE-based scheme. The PMCE method has been demonstrated to be a suitable technique for the design of 2-D optical ZRSs that are robust against diffraction effects.

\section{REFERENCES}

[1] Y. Chen, W. Huang, and X. Dang, "Design and analysis of two dimensional zero-reference marks for alignment systems," Rev. Sci. Instrum., vol. 74, no. 7, pp. 3549-3553, 2003.

[2] J. Sáez-Landete, J. Alonso, and E. Bernabeu, "Design of twodimensional zero reference codes by means of a global optimization method," Opt. Express, vol. 13, no. 11, pp. 4230-4236, 2005.

[3] J. Sáez-Landete, S. Salcedo-Sanz, M. Rosa-Zurera, J. Eusebio, and A. Bernabeu, "Design of two-dimensional zero reference codes with a genetic algorithm," Opt. Lett., vol. 31, no. 11, pp. 1648-1650, 2006.

[4] J. Chen, "Design of two-dimensional zero reference codes with crossentropy method," Appl. Opt., vol. 49, no. 18, pp. 3560-3565, 2010.

[5] Y. Su, T. Wu, C. Wang, and M. Chang, "Generation of two-dimensional optical reference signals based on parametric minimum cross entropy,' IEEE Photon. Technol. Lett., vol. 23, no. 12, pp. 813-815, 2011.

[6] C. Zhou, Y. Wang, Y. Chen, and W. Huang, "Alignment measurement of two-dimensional zero-reference marks," Precis. Eng., vol. 30, no. 2, pp. 238-241, 2006.

[7] J. Sáez-Landete, S. Salcedo-Sanz, F. Cruz-Roldán, P. Amo-López, and M. Blanco-Velasco, "Design of two-dimensional optical alignment signals robust to diffractive effects," J. Lightwave Technol., vol. 26, no. 12, pp. 1702-1707, 2008.

[8] J. W. Goodman, Introduction to Fourier Optics. New York: McGraw-Hill Science, 1996.

[9] J. Chen, S. Wang, M. Lee, and C. Li, "Cross-entropy method for the optimization of optical alignment signals with diffractive effects," $J$. Lightwave Technol., vol. 29, no. 18, pp. 2706-2714, 2011.

[10] R. Y. Rubinstein and A. Dolgin, "Fast parametric entropy-based and the parametric MinxEnt method for counting of \#P-complete problems," private communication, 2007. 